\title{
A Novel Method of Constructing Human Middle Finger from its Fractional Part
}

\author{
C.N. Ravi Kumar \\ SJCE \\ Mysore, 570006, India
}

\author{
Manimala.S \\ SJCE \\ Mysore, 570006, India
}

\begin{abstract}
The human hand is a masterpiece of mechanical complexity. The anatomy of the hand is complex, intricate, and fascinating. Its integrity is absolutely essential for everyday functional living. Hands may be affected by many disorders, most commonly traumatic injury. In treating hand problems, the mastery of anatomy is fundamental in order to provide the best quality of care. In this paper, the focus is on the construction of middle finger of the hand when only fractional part of the finger tip image is available. 24 Geometric features of both the hands from 100 people of different age group were extracted from the silhouettes. From the known middle finger width, the proposed method can be used to estimate middle finger length, position of knuckles and also finger width at the second knuckle using taalamana system and shilpa shastra. 600 images of various age groups were collected and from the fractional part of the middle finger image, complete middle finger is constructed based on the estimated values of finger length and width. The estimation accuracy of more than $91 \%$ is achieved for all the estimated features. Our construction algorithm efficiently works for various cases like fingers with nail, fingers of left or right hand and fingers with high degree of variation in color and age.
\end{abstract}

\section{General Terms}

Image Processing, Image Construction

\section{Keywords}

finger construction, golden mean, taalamana system, extrapolation, iconography.

\section{INTRODUCTION}

The hands play an important part in non-verbal communication. Hands often approach the face during social interaction. Beauty consciousness among people is increasing, particularly in women. Hence the need for cosmetic surgery has increased. Due to its affordability people are interested in the beauty of their hands as well. Cosmetic surgeons use computers to gain prior knowledge about the exact proportions of the hand before the actual surgery.

Reconstructive plastic surgery is often performed on burn and accident victims. It may involve the rebuilding of severely fractured bones, as well as skin grafting. Reconstructive surgery includes such procedures as the reattachment of an amputated finger or toe. Number of road, rail and air accidents is increasing. Human ability to transplant organs and reconstruction of lost parts of the human body is also increasing. So also the computing facility is becoming more cost effective. In case of accidents, if only partial knowledge of the finger is available, then the proposed method can be used to obtain complete knowledge and reconstruct the damaged part to its original form.

Construction of the middle finger when only fractional part of the image is available is a challenging task. The task is achieved using taalamana system and golden mean.

\subsection{Taalamana System}

Iconography is the branch of art history which studies the identification, description, and the interpretation of the content of images. The word iconography literally means "image writing". The idea of constructing human hand is derived from Shilpa Shastra. It has developed its own norms of measures and proportions. It is a complex system of iconography that defines rigid definitions $[1,21,22]$. The shilpa shastra normally employ divisions on a scale of one (eka tala) to ten (dasa tala). Each tala is subdivided into 12 angulas. It is called Taalamana paddathi or Taalamana system, the system of measurements by Tala, the palm of hand i.e. from the tip of the middle finger to the wrist as shown in figure 1.

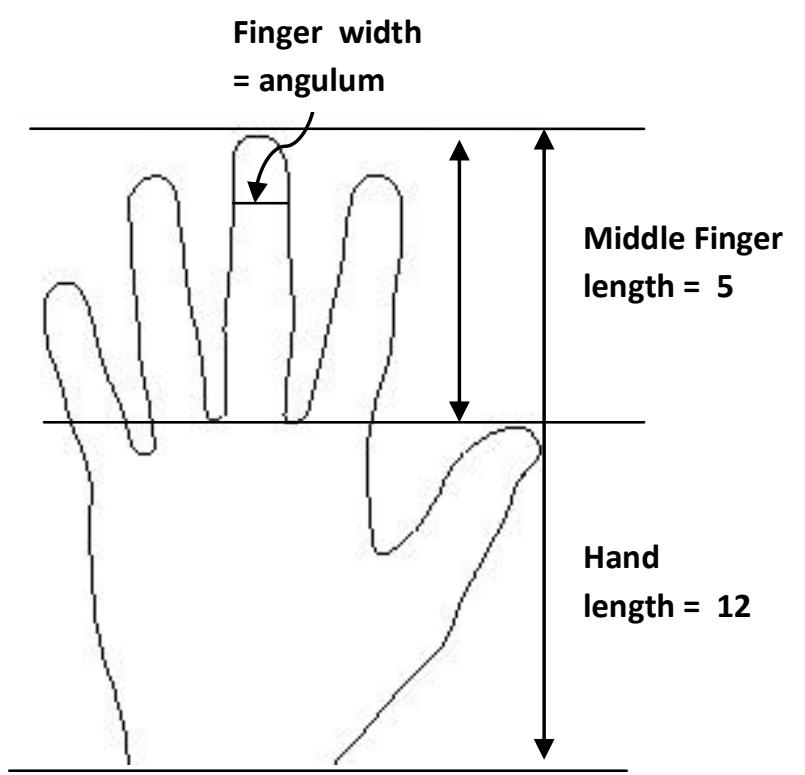

Figure 1: Computation of Middle finger length

\subsection{Golden ratio}

Two quantities are in the golden ratio if the ratio of the sum of the quantities to the larger quantity is equal to the ratio of the larger quantity to the smaller one. The golden section is a line segment divided according to the golden ratio. If $\mathrm{a}$ and $\mathrm{b}$ are the 
lengths of the larger and smaller line segments respectively, then golden ratio is represented as shown in equation 1 .

$$
\frac{a+b}{a}=\frac{a}{b}=\Phi(P h i)
$$

The work is presented in five sections. Introduction is given in the first section. In the second section literature survey is presented. Mathematical model is enumerated in section 3. In section 4 the proposed method is discussed and the simulation results are presented in section 5 .

\section{LITERATURE SURVEY}

Geometric measurements of the human hand have been used for identity authentication in a number of commercial systems. Anil K. Jain and others have worked extensively on hand geometry specifically for identification and verification systems $[6,7,8]$. There is not much open literature addressing the research issues underlying hand geometry-based identity authentication; much of the literature is in the form of patents [2, 3, 4]. Hand geometry recognition systems may provide three kinds of services like verification, classification and identification [12]. A novel contact-free biometric identification system based on geometrical features of the human hand is developed by Aythami Morales and others [11]. A component-based hand verification system using palm-finger segmentation and fusion was developed by Gholamreza and others. The geometry of each component of the hand is represented using high order Zernike moments which is computed using an efficient methodology [15].

Windy and others have used geometric measurements to study the sexual orientation. The ratio of the length of the second digit (2D) to the length of the fourth digit (4D) is greater in women than in men. This ratio is stable from 2 years of age in humans $[9,10]$. Gender classification from hand images in computer vision is attempted by Gholamreza and others [16].

Issac Cohen and others have worked on 3D hand construction from silhouettes of 2D hands [13]. Digital and metacarpal formulae are morphological variables which may also have functional significance in the understanding of how certain hand forms may be ill-fitted for certain tasks [14].

T.F.Cootes and others have worked on active shape models $[17,18]$ which laid foundations for statistical shape analysis using Procrustes analysis, tangent space projection and Principal Component Analysis[19]. Geometric hand measurements are also used in hand gesture classification using a view-based approach for representation and Artificial Neural Network for classification [20].

\section{MATHEMATICAL MODEL}

Prediction of finger length, position of knuckles and finger width at the second knuckle are computed using taalamana system and golden ratio. The finger length is computed as five times the finger width (FW1). Finger joints or knuckles are estimated using golden ratio rule. The golden mean or ratio can be computed mathematically as shown in equation 2 and 3 .

$$
\begin{aligned}
& \frac{\sqrt{5}+1}{2}=\Phi(P h i)=1.6180339 . . \\
& \frac{\sqrt{5}-1}{2}=\Phi(p h i)=0.6180339 . .
\end{aligned}
$$

Positions of the knuckles from tip and bottom of the finger are computed using the equation 4 and the finger width at the second knuckle FW2 is computed as shown in equation 5.

$$
L 1=L 2=p h i * F L \ldots \ldots \ldots
$$

$F W 2=2 * F W 1-(p h i * F W 1) \ldots \ldots \ldots \ldots$

\section{PROPOSED METHOD}

Silhouettes of both the hands of 100 users are taken, features are extracted as described in section 4.1. Using the proposed method the feature values are estimated and compared with that of the actual values obtained. Using digital camera three images of each hand is captured. A hand database of 600 images is created. Construction of middle finger is discussed in section 4.2.

\subsection{Estimation of Finger Length, Position of Knuckles from Known Finger Width(FW1)}

Geometric features of both the hands from 100 users of different age group are collected. At first the silhouette of hand is taken on the paper and the positions of the knuckles are marked. 24 features are extracted as shown in figure 2 . For each finger five features namely finger width 1 (FW1), finger width 2 (FW2), Finger Length (FL), Position of first knuckle from bottom(L1) and position of second knuckle from finger tip (L2) are extracted. Similarly for the four fingers these five features are collected and four features for the thumb totally to 24 feature set. From first width of the middle finger (FW1), the values of FL, L1, L2 and FW2 are estimated. The actual and estimated values of a subset of 25 samples are tabulated in Table 1 and Table 2 in page 5 .

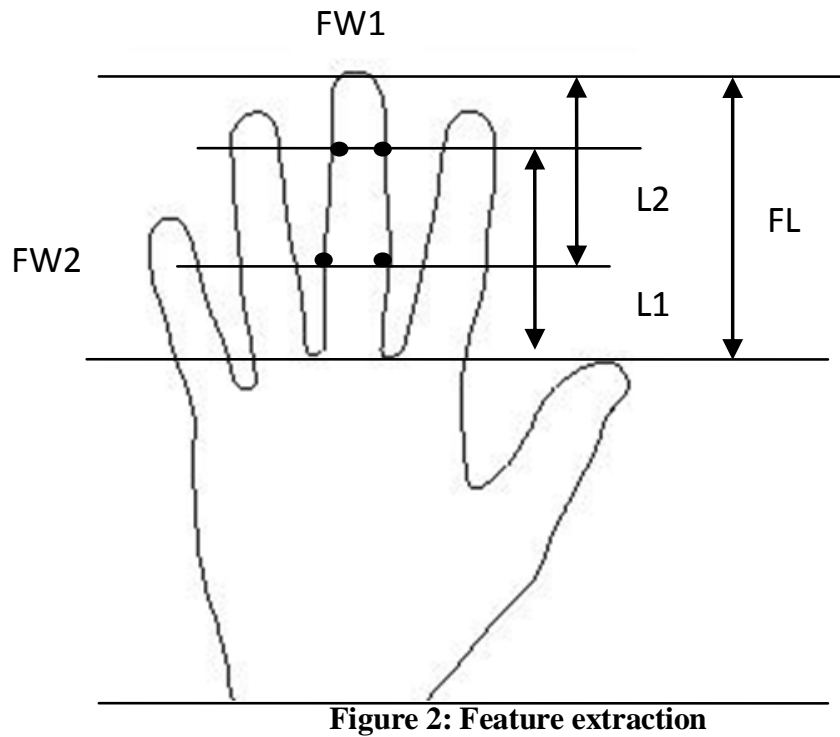


Table 1: Actual and estimated values of FL and FW2

\begin{tabular}{|l|l|l|l|l|l|l|l|l|l|}
\hline $\begin{array}{l}\text { Sl. } \\
\text { No. }\end{array}$ & FW1 & $\begin{array}{l}\text { A- } \\
\text { FL }\end{array}$ & $\begin{array}{l}\text { PL } \\
\text { FL }\end{array}$ & $\begin{array}{l}\text { Abs } \\
\text { Error }\end{array}$ & \%Correct & $\begin{array}{l}\text { A- } \\
\text { FW2 }\end{array}$ & $\begin{array}{l}\text { P- } \\
\text { FW2 }\end{array}$ & $\begin{array}{l}\text { Abs } \\
\text { Error }\end{array}$ & \% Correct \\
\hline 1 & 1.5 & 7.5 & 7.5 & 0 & 100 & 1.9 & 1.809 & 0.091 & 94.97 \\
\hline 2 & 1.6 & 8 & 8 & 0 & 100 & 2 & 1.9296 & 0.0704 & 96.352 \\
\hline 3 & 1.4 & 7 & 7 & 0 & 100 & 1.75 & 1.6884 & 0.0616 & 96.352 \\
\hline 4 & 1.4 & 7 & 7 & 0 & 100 & 1.8 & 1.6884 & 0.1116 & 93.39 \\
\hline 5 & 1.7 & 8 & 8.5 & 0.5 & 94.118 & 2 & 2.0502 & 0.0502 & 97.551 \\
\hline 6 & 1.7 & 8.1 & 8.5 & 0.4 & 95.294 & 2 & 2.0502 & 0.0502 & 97.551 \\
\hline 7 & 1.42 & 7.2 & 7.1 & 0.1 & 98.592 & 1.8 & 1.7125 & 0.0874 & 94.892 \\
\hline 8 & 1.4 & 6.8 & 7 & 0.2 & 97.143 & 1.8 & 1.6884 & 0.1116 & 93.39 \\
\hline 9 & 1.4 & 7.1 & 7 & 0.1 & 98.571 & 1.6 & 1.6884 & 0.0884 & 94.764 \\
\hline 10 & 1.4 & 7 & 7 & 0 & 100 & 1.9 & 1.6884 & 0.2116 & 87.467 \\
\hline 11 & 1.5 & 7.7 & 7.5 & 0.2 & 97.333 & 1.8 & 1.809 & 0.009 & 99.502 \\
\hline 12 & 1.6 & 7.9 & 8 & 0.1 & 98.75 & 2 & 1.9296 & 0.0704 & 96.352 \\
\hline 13 & 1.5 & 7.2 & 7.5 & 0.3 & 96 & 2 & 1.809 & 0.191 & 89.442 \\
\hline 14 & 1.6 & 7.5 & 8 & 0.5 & 93.75 & 2 & 1.9296 & 0.0704 & 96.352 \\
\hline 15 & 1.6 & 7.7 & 8 & 0.3 & 96.25 & 1.9 & 1.9296 & 0.0296 & 98.466 \\
\hline 16 & 1.5 & 7.4 & 7.5 & 0.1 & 98.667 & 1.9 & 1.809 & 0.091 & 94.97 \\
\hline 17 & 1.4 & 7.4 & 7 & 0.4 & 94.286 & 1.8 & 1.6884 & 0.1116 & 93.39 \\
\hline 18 & 1.4 & 7.4 & 7 & 0.4 & 94.286 & 1.8 & 1.6884 & 0.1116 & 93.39 \\
\hline 19 & 1.6 & 8.1 & 8 & 0.1 & 98.75 & 1.9 & 1.9296 & 0.0296 & 98.466 \\
\hline 20 & 1.7 & 8.3 & 8.5 & 0.2 & 97.647 & 2.1 & 2.0502 & 0.0498 & 97.571 \\
\hline 21 & 1.9 & 8.8 & 9.5 & 0.7 & 92.632 & 2.3 & 2.2914 & 0.0086 & 99.625 \\
\hline 22 & 2 & 9.2 & 10 & 0.8 & 92 & 2.5 & 2.412 & 0.088 & 96.352 \\
\hline 23 & 1.8 & 9 & 9 & 0 & 100 & 2.2 & 2.1708 & 0.0292 & 98.655 \\
\hline 24 & 1.8 & 9.1 & 9 & 0.1 & 98.889 & 2.3 & 2.1708 & 0.1292 & 94.048 \\
\hline 25 & 1.7 & 8.2 & 8.5 & 0.3 & 96.471 & 2 & 2.0502 & 0.0502 & 97.551 \\
\hline
\end{tabular}

Table 2: Actual and estimated values of $\mathrm{L1}$ and L2

\begin{tabular}{|l|l|l|l|l|l|l|l|l|l|}
\hline $\begin{array}{l}\text { Sl. } \\
\text { No. }\end{array}$ & FW1 & $\begin{array}{l}\text { A- } \\
\text { L1 }\end{array}$ & P-L1 & $\begin{array}{l}\text { Abs } \\
\text { Error }\end{array}$ & \%Correct & $\begin{array}{l}\text { A- } \\
\text { L2 }\end{array}$ & P-L2 & $\begin{array}{l}\text { Abs } \\
\text { Error }\end{array}$ & \% Correct \\
\hline 1 & 1.5 & 4.9 & 5.385 & 0.485 & 90.994 & 4.9 & 4.635 & 0.265 & 94.283 \\
\hline 2 & 1.6 & 5.6 & 5.744 & 0.144 & 97.493 & 5.1 & 4.944 & 0.156 & 96.845 \\
\hline 3 & 1.4 & 4.6 & 5.026 & 0.426 & 91.524 & 4.5 & 4.326 & 0.174 & 95.978 \\
\hline 4 & 1.4 & 5.1 & 5.026 & 0.074 & 98.528 & 4.8 & 4.326 & 0.474 & 89.043 \\
\hline 5 & 1.7 & 6 & 6.103 & 0.103 & 98.312 & 5.5 & 5.253 & 0.247 & 95.298 \\
\hline 6 & 1.7 & 5.5 & 6.103 & 0.603 & 90.12 & 5.2 & 5.253 & 0.053 & 98.991 \\
\hline 7 & 1.42 & 5 & 5.0978 & 0.0978 & 98.082 & 4.2 & 4.3878 & 0.1878 & 95.72 \\
\hline 8 & 1.4 & 4.4 & 5.026 & 0.626 & 87.545 & 4.4 & 4.326 & 0.074 & 98.289 \\
\hline 9 & 1.4 & 4.8 & 5.026 & 0.226 & 95.503 & 4.5 & 4.326 & 0.174 & 95.978 \\
\hline 10 & 1.4 & 4.8 & 5.026 & 0.226 & 95.503 & 4.8 & 4.326 & 0.474 & 89.043 \\
\hline 11 & 1.5 & 5.4 & 5.385 & 0.015 & 99.721 & 4.8 & 4.635 & 0.165 & 96.44 \\
\hline 12 & 1.6 & 5.2 & 5.744 & 0.544 & 90.529 & 5.4 & 4.944 & 0.456 & 90.777 \\
\hline 13 & 1.5 & 4.8 & 5.385 & 0.585 & 89.136 & 4.8 & 4.635 & 0.165 & 96.44 \\
\hline 14 & 1.6 & 5 & 5.744 & 0.744 & 87.047 & 5 & 4.944 & 0.056 & 98.867 \\
\hline 15 & 1.6 & 5 & 5.744 & 0.744 & 87.047 & 5 & 4.944 & 0.056 & 98.867 \\
\hline 16 & 1.5 & 4.9 & 5.385 & 0.485 & 90.994 & 4.7 & 4.635 & 0.065 & 98.598 \\
\hline 17 & 1.4 & 5.4 & 5.026 & 0.374 & 92.559 & 5 & 4.326 & 0.674 & 84.42 \\
\hline 18 & 1.4 & 5.2 & 5.026 & 0.174 & 96.538 & 4.7 & 4.326 & 0.374 & 91.355 \\
\hline 19 & 1.6 & 5.3 & 5.744 & 0.444 & 92.27 & 5.3 & 4.944 & 0.356 & 92.799 \\
\hline 20 & 1.7 & 5.5 & 6.103 & 0.603 & 90.12 & 5.4 & 5.253 & 0.147 & 97.202 \\
\hline 21 & 1.7 & 5.6 & 6.103 & 0.503 & 91.758 & 6 & 5.253 & 0.747 & 85.78 \\
\hline 22 & 2 & 6.2 & 7.18 & 0.98 & 86.351 & 5.8 & 6.18 & 0.38 & 93.851 \\
\hline 23 & 1.8 & 6 & 6.462 & 0.462 & 92.851 & 5.7 & 5.562 & 0.138 & 97.519 \\
\hline 24 & 1.8 & 6.2 & 6.462 & 0.262 & 95.946 & 5.6 & 5.562 & 0.038 & 99.317 \\
\hline 25 & 1.7 & 5.8 & 6.103 & 0.303 & 95.035 & 5.1 & 5.253 & 0.153 & 97.087 \\
\hline
\end{tabular}




\subsection{Construction of Middle Finger}

The color image of the entire palm is captured of size $640 \times 480$ and is in jpg format. Part of the middle finger is extracted manually from the palm image. Read the available part of the finger image and preprocess the color image to convert into binary image. Compute the finger width (FW1) from the available part of the finger image also called as an angulum. Based on taalamana system, compute the finger length to be equal to five times to that of the finger width. Extrapolate the pixels in the available image upto the computed finger length. The positions of the two knuckles are computed using equation 4.

\section{SIMULATION RESULTS}

\subsection{Estimation of Middle Finger Features}

24 geometrical features of both the hands are collected from 100 different people of different age group. Features collected for each of the finger are Finger Width (FW1, FW2), Finger Length (FL), Distance of first knuckle from bottom of the finger (L1) and distance of the second knuckle from the tip of the finger (L2). Total of 24 features are extracted. In the current study only middle finger features are computed using our approach.

Mean Square Error or MSE of an estimator quantifies the difference between an estimator and the true value of the quantity being estimated. MSE measures the average of the square of the "error." The error is the amount by which the estimator differs from the quantity to be estimated. The square root of MSE yields the root mean squared error or RMSE.

$$
\begin{aligned}
& M S E=\frac{1}{n} \sum_{i=1}^{k} f_{i}-y_{i}^{2} \\
& M A E=\frac{1}{n} \sum_{i=1}^{k} a b s \quad f_{i}-y_{i}
\end{aligned}
$$

The Mean Absolute Error (MAE) measures how close forecasts or predictions are to the eventual outcomes. MAE is an average of the absolute errors computed as in equation 7, where fi is the estimated value and yi the true value.

Table 1 tabulates the actual and estimated values of FW1, FL and FW2. The finger length is estimated as five times of FW1. Finger width 2 is estimated using the equation 5. Absolute error and percentage of correctness for both FL and FW2 are also tabulated. Only 25 random samples are shown in the table. Similarly, in Table 2 the actual and positions of first knuckle (L1) and second knuckle (L2) are tabulated along with the absolute error. The statistical features of the samples namely min, max, mean and standard deviation are tabulated in Table 3. Table 4 shows RMSE, MAE and estimation accuracy of all the four features estimated for the middle finger. Mean absolute error and Root mean square error tabulated indicates that a maximum of 0.4 centimeters error is present in estimating position of the knuckles and approximately 0.39 centimeters in estimating finger length.

Table 3 : Statistical Analysis

\begin{tabular}{|l|l|l|l|c|}
\hline & Min & Max & Mean & Std Deviation \\
\hline FW1 & 1.3 & 2.0 & 1.5712 & 0.1246 \\
\hline FL & 6.8000 & 9.6000 & 8.0374 & 0.5418 \\
\hline FW2 & 1.4000 & 2.5000 & 1.7582 & 0.2013 \\
\hline L1 & 4.4000 & 6.6000 & 5.5226 & 0.4200 \\
\hline L2 & 4.2000 & 6.8000 & 5.2632 & 0.4563 \\
\hline
\end{tabular}

Table 4 : RMSE and MAE

\begin{tabular}{|l|c|c|c|}
\hline & MAE & RMSE & $\begin{array}{c}\text { Estimation } \\
\text { Accuracy }\end{array}$ \\
\hline FL & 0.3010 & 0.39881 & $96.03 \%$ \\
\hline FW2 & 0.2013 & 0.18908 & $91.13 \%$ \\
\hline L1 & 0.4199 & 0.42848 & $94.17 \%$ \\
\hline L2 & 0.4563 & 0.51219 & $91.14 \%$ \\
\hline
\end{tabular}

In figure 3(a-d) shown in page 7, around $40-50$ subset of the samples are plotted which indicates the actual and estimated values of FL, FW2, L1 and L2 respectively.

Red line in the plot shows the actual or true values and blue line indicates the estimated values. Overlapping in the graph shows the close relation of estimated values to the actual values.

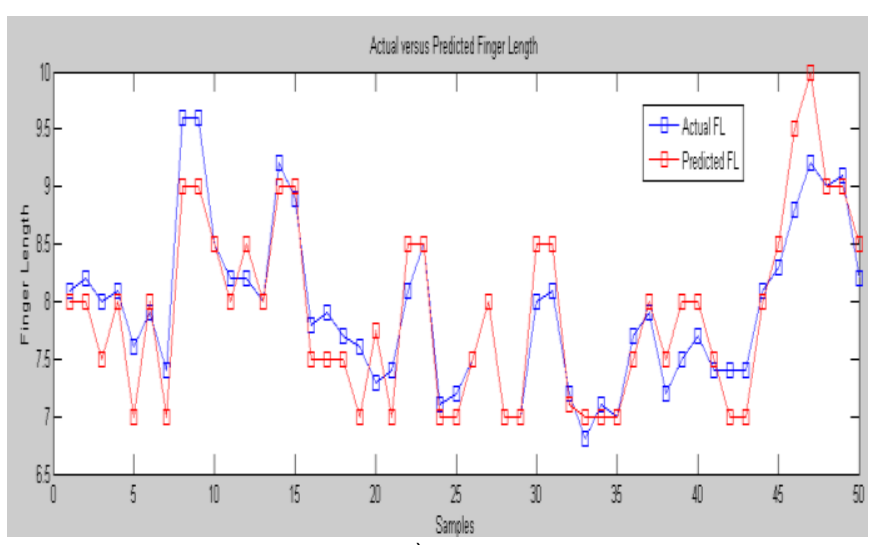

a)

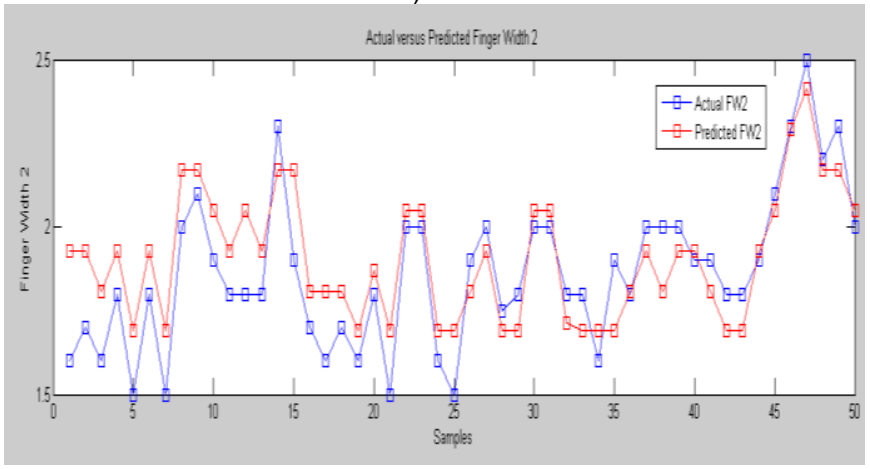

b) 


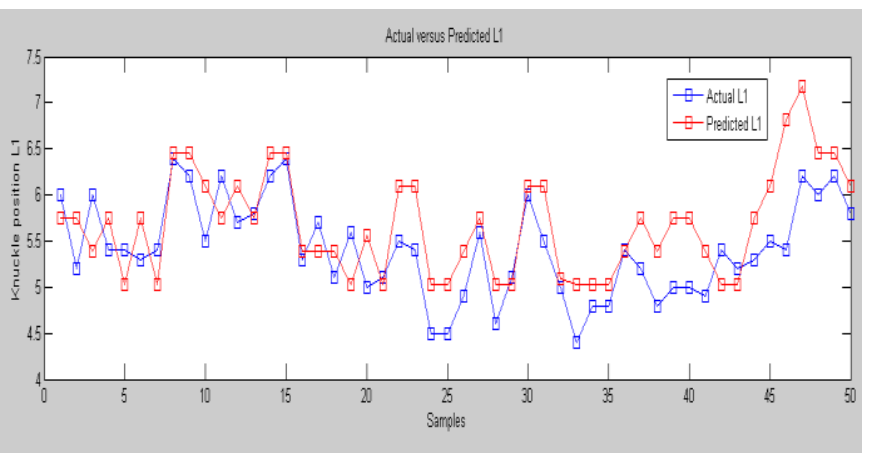

C)

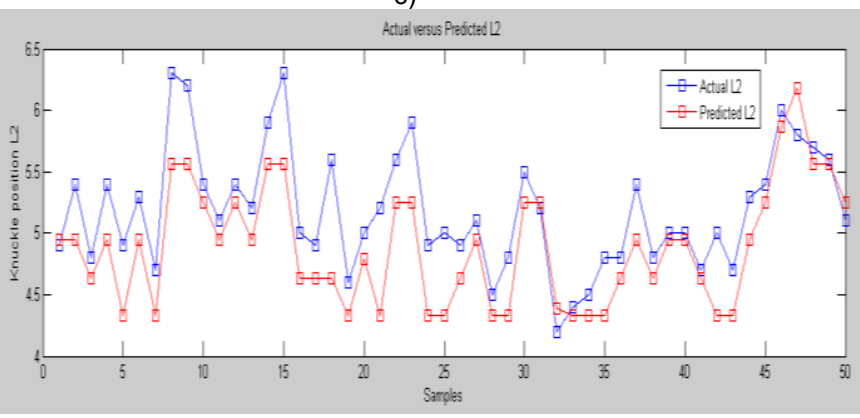

d)

Figure 3(a-d) : Plot of actual and estimated values of FL, FW2, $\mathrm{L} 1$ and $\mathrm{L} 2$

\subsection{Construction of Finger from its Fractional Part}

From the digital image of the entire palm a part of the middle finger is manually extracted. The size of the fractional image is not fixed. Figure $4 \mathrm{a}$ to $4 \mathrm{~d}$ shows the process of construction of middle finger from its fractional image.
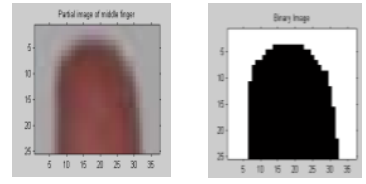

$\begin{array}{cc}\text { a)Partial } & \text { b) Binary } \\ \text { Finger } & \text { Image }\end{array}$

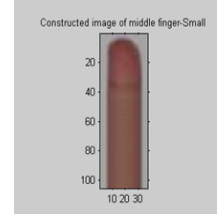

c) Constructed Finger

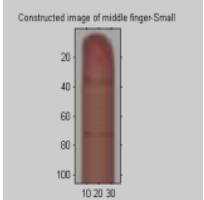

d) Constructed Finger with finger joints
Figure 4 : Construction of Middle Finger

The given image is converted into binary image and finger width (FW1) feature is extracted from it. Using the method discussed in section 4.1, the values for FL, L1, L2 and FW2 are estimated. In figure 4 , ' $a$ ' shows the partial image of the finger, ' $b$ ' shows its binary representation, ' $c$ ' shows the constructed image of the finger and ' $\mathrm{d}$ ' indicates image of the middle finger with positions two knuckles marked. Figure 5 shows a set of six samples of fractional middle finger images named ' $a$ ' through ' $f$ '. Our approach efficiently works for various cases like fingers with nail, finger with high degree of variation in color and age.
Also works on male and female fingers. Figure $6 a$ and $6 \mathrm{~b}$ indicates the construction of the middle finger for the six samples shown in figure 5 .
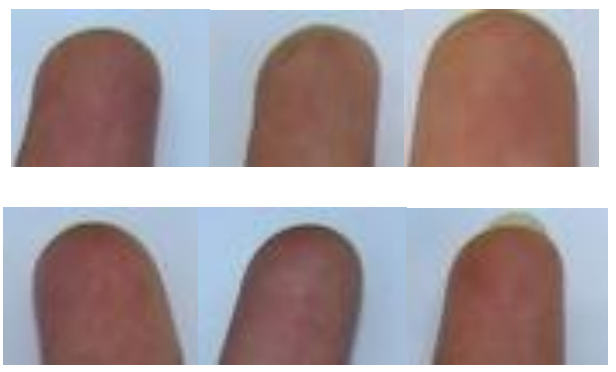

Figure 5(a-f) : Six samples of fractional middle Finger images

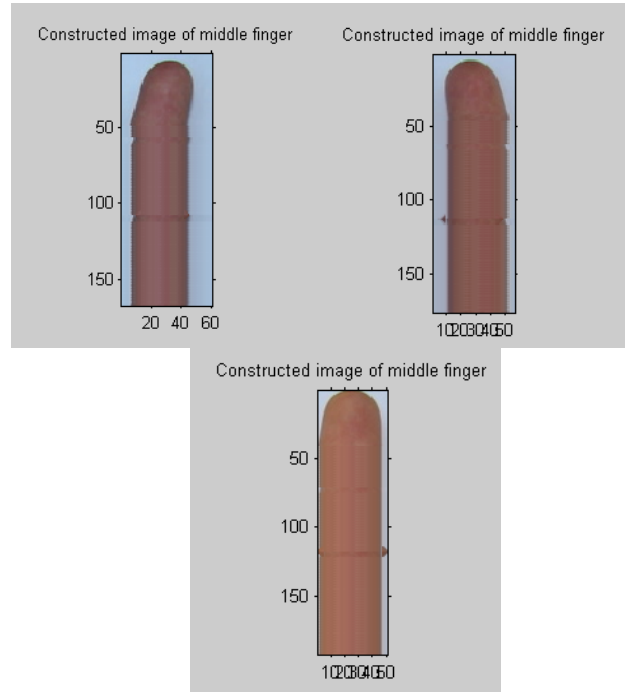

Figure 6a : Constructed finger from a-c of sample image

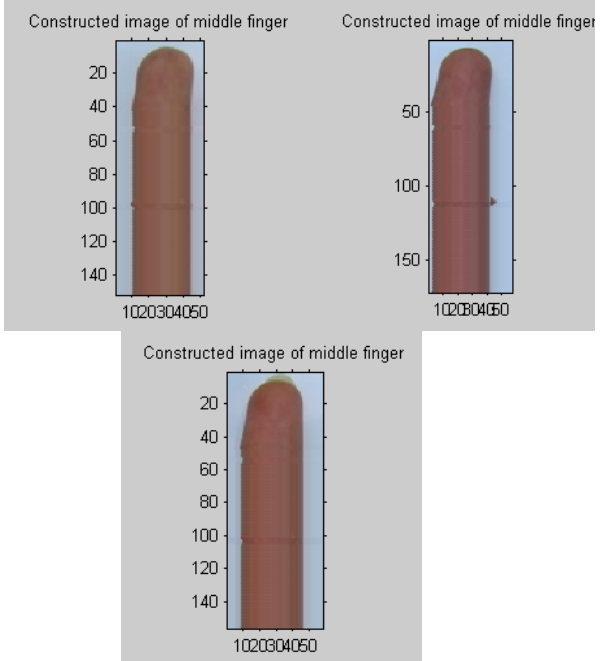

Figure 6b : Constructed finger from d-f of sample image 


\section{CONCLUSION}

To the best of our knowledge this is the first humble beginning in constructing human finger from its fractional part. Taalamana system and golden ratio are used to predict the feature values for FL, FW2, L1 and L2. The graph in figure 3 indicates close association of the actual and the estimated feature values. Estimation accuracy of $96 \%, 91 \%, 94 \%$ and $91 \%$ for FL, FW2, L1 and L2 features respectively is achieved. Middle finger is constructed using the estimated values of the features and the available fractional image of the finger. The proposed construction algorithm can be efficiently applied of diverse samples including high variation in color and age, male or female and left or right hand finger.

\section{REFERENCES}

[1] Gopinatha Rao, T. A (1920). Talamana, or, Iconometry : Memoirs of the Archaeological Survey of India; no. 3. Calcutta: Supt. Govt. Print

[2] R. P. Miller, "Finger dimension comparison identification system", US Patent No. 3576538, 1971.

[3] R. H. Ernst, "Hand ID system", US Patent No.3576537, 1971.

[4] H. Jacoby, A. J. Giordano, and W. H. Fioretti, "Personnel Identification Apparatus", US PatentNo. 3648240, 1972.

[5] Raul Sanchez -Reillo, Carmen Sanchez-Avila, Ana Gonzalez-Macros, "Biometric identification through hand geometric measurements", IEEE Transactions on pattern analysis and machine intelligence, Vol 22, No. 10, Oct 2000.

[6] Anil K. Jain, Arun Ross, Sharath Pankanti, "A Prototype Hand Geometry-based Verification System", 2nd International Conference on Audio- and Video-based Biometric Person Authentication (AVBPA), Washington D.C., pp.166-171, March 22-24, 1999.

[7] A.K. Jain, A. Ross, and S. Pankanti. A prototype hand geometrybased verification system. In Proceedings of 2nd Int'l Conference on Audio- and Video-based Biometric Person Authentication, pages 166-171, March 1999.

[8] Anil K. Jain and Nicolae Duta. Deformable matching of hand shapes for verification. In Proceedings of International Conference on Image Processing, October 1999.

[9] Windy M. Brown, Melissa Hines, Briony A. Fane, S. Marc Breedlove, " Masculinized Finger Length Patterns in Human Males and Females with Congenital Adrenal Hyperplasia", Hormones and Behavior 42, 380-386 2002, Elsevier Science (USA)

[10] Windy M. Brown, B.A., Christopher J. Finn, B.A., Bradley M. Cooke, and S. Marc Breedlove,"Differences in Finger Length Ratios Between Self-Identified Butch and Femme Lesbians", Archives of Sexual Behavior, Vol. 31, No. 1, February 2002, pp. 123-127
[11] Aythami Morales, Miguel A. Ferrer, Francisco Díaz, Jesús B. Alonso, Carlos M. Travieso, "Contact-free hand biometric system for real environments".

[12] Yaroslav Bulatov, Sachin Jambawalikar, Piyush Kumar, Saurabh Sethia, "Hand recognition using geometric classifiers".

[13] Isaac Cohen, Sung Uk Lee , "3D Hand and Fingers Reconstruction from Monocular View"

[14] Stephen Lewis "Morphological aspects of male and female hands"

[15] Gholamreza Amayeh, George Bebis, Ali Erol, Mircea Nicolescu, "A Component-Based Approach to Hand Verification",

[16] Gholamreza Amayeh, George Bebis, Mircea Nicolescu, "Gender Classification from Hand Shape",

[17] T. F. Cootes and Taylor, "Active shape models - smart snakes", British Machine Vision Conference, pages 266$275,1992$.

[18] T. F. Cootes, G. J. Edwards, and C. J. Taylor, "Active appearance models", IEEE Transactions On Pattern Recognition and Machine Intelligence, 23(6):681-685, 2001.

[19] Mikkel B. Stegmann and David Delgado Gomez, "A Brief Introduction to Statistical Shape Analysis", a data report at http://www.imm.dtu.dk/ mbs/

[20] Sanjay Kumar, Dinesh K Kumar, Arun Sharma, and Neil McLachlan "Classification of Hand Movements Using Motion Templates and Geometrical Based Moments", IEEE, ICISIP 2004

[21] Gift Siromoney; M. Bagavandas, S.Govindaraju (1980). "An iconometric study of Pallava sculptures". Kalakshetra Quarterly 3 (2): 7-15.

[22] http://www.cmi.ac.in/gift/Iconometry/icon_pallavasculptur e.htm

[23] Kramrisch, Stella; Raymond Burnier (1976). The Hindu Temple. Motilal Banarsidass Publ.. pp. 309. ISBN 9788120802247.

[24] Wangu, Madhu Bazaz. Images of Indian Goddesses: Myths, Meanings and Models. Abhinav Publications. pp. 72. ISBN 9788170174165

[25] Manimala.S, Dr. C N Ravi Kumar," Prediction of Middle Finger Features from its Width: A Novel Approach", International Journal of Advanced Research in Computer Science, Vol 1, Issue 4, 2010, ISSN :09765697,pp 42-46 